\title{
Endoscopic submucosal dissection of a giant esophageal lymphangioma
}
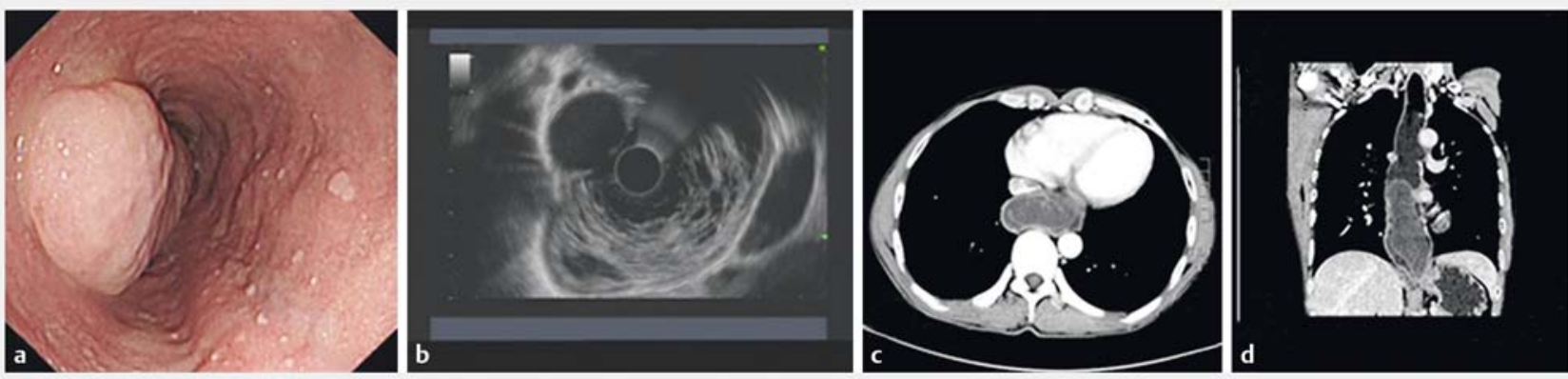

- Fig. 1 a Esophagogastroduodenoscopy found a giant, yellowish, and translucent submucosal tumor, with a lustrous surface and vesicular change. b Endoscopic ultrasound showed a grid-like hypoechoic structure with a heterogeneous echo that originated from the submucosal layer; the muscularis propria was intact. c, $\mathbf{d}$ Contrast-enhanced chest computed tomography revealed an irregular, intraluminal, and low-density mass $(16 \times 6 \times 4 \mathrm{~cm})$ in the middle-to-lower part of the esophagus.

Lymphangioma of the esophagus is an exceedingly rare submucosal benign tumor [1]. Only 23 cases have been reported since 1934, and most of the tumors are smaller than $2 \mathrm{~cm}$ in diameter. Herein, we present the first report of a giant esophageal lymphangioma (16× $6 \times 4 \mathrm{~cm}$ ), which was completely and safely resected by endoscopic submucosal dissection (ESD).

A 46-year-old man suffering dysphagia for 3 years visited our department. Computed tomography revealed an irregular, intraluminal, and low-density mass (16x $6 \times 4 \mathrm{~cm})$ in the middle-to-lower part of the esophagus ( $\mathbf{F i g . 1} \mathbf{c}, \mathbf{d}$ ). Esophagogastroduodenoscopy revealed a giant, yellowish, and translucent submucosal lesion, with a lustrous surface and vesicular change in the esophagus at $28 \mathrm{~cm}$ from the incisor and extending into the lesser curvature of the cardia ( $\vee$ Fig. 1 a). Endoscopic ultrasonography showed a grid-like hypoechoic structure with heterogeneous echo that originated from the submucosal layer without involving the muscle layer ( $\triangleright$ Fig. $\mathbf{1} \mathbf{b}$ ).

ESD was performed to relieve the patient's symptoms ( $\bullet$ Video 1$)$. First, circumferential marking was made using a dual knife ( $\vee$ Fig. $\mathbf{2 a}$ ). Then, a solution of indigo carmine and glycerol was injected to lift the submucosa ( $\triangleright$ Fig.2 $\mathbf{b}$ ). After circumferential mucosal incision,

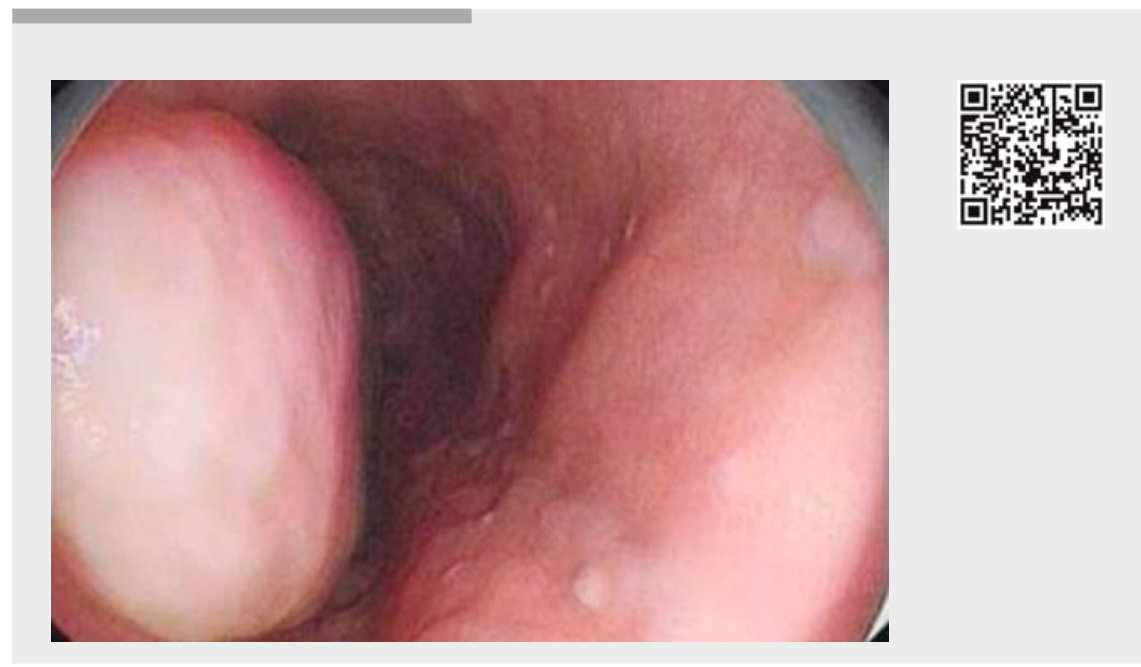

Video 1 Endoscopic submucosal dissection of a giant esophageal lymphangioma.

a clip with dental floss was placed directly onto the dissected submucosal tissue, which provided proper countertraction for a clear submucosal dissection plane during submucosal dissection ( $\mathbf{F i g . 2 c , d , e , g ) . ~ T h e ~ t u m o r ~ w a s ~ c o m - ~}$ pletely and uneventfully resected en bloc; however, because of its large size, we could not retrieve it en bloc, even under the vigorous support of specialist doctors. Given the benign nature of the tumor, it was finally divided into pieces in the esophagus and subsequently retrieved using a snare ( $\bullet$ Fig. $\mathbf{2 f}$, $\triangleright$ Fig. $\mathbf{3}$ ).
Histologically, the tumor showed irregularly dilated lymphatic vessels beneath squamous epithelium and the submucosa, with lymphoid fluid in the lumen ( $\vee$ Fig.4a,b), and accompanying CD31

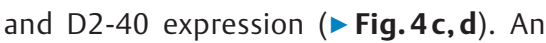
esophageal lymphangioma was diagnosed.

At 2 weeks after surgery, triamcinolone acetonide (Kenacort, $40 \mathrm{mg} / \mathrm{mL}$; Zhejiang Xianju Pharmaceutical Co., Ltd. Zhejiang, China), diluted 1:9 with saline, was injected at several points in the submucosa to prevent esophageal stenosis. 

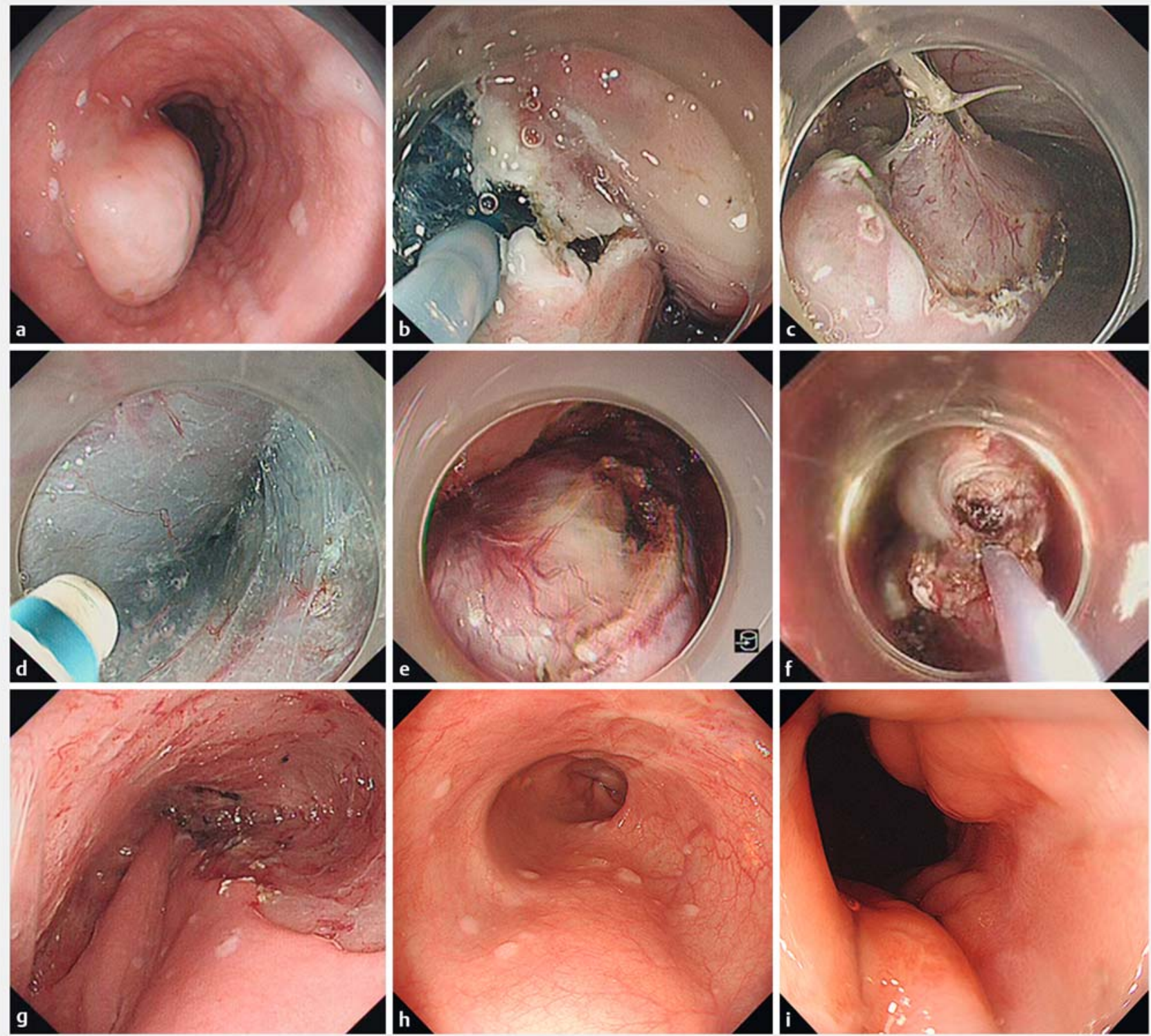

- Fig. 2 Endoscopic submucosal dissection (ESD) procedure. a The resection borders were marked. b A solution of indigo carmine and glycerol was injected to lift the submucosa. The mucosa was cut around the lesion. c A clip with dental floss provided traction. $\mathbf{d}$ The lesion was quickly peeled away using an insulation-tipped knife. $\mathbf{e}$ The tumor was resected en bloc. $\mathbf{f}$ The tumor was divided into smaller pieces and retrieved from the esophagus using a snare. $\mathbf{g}$ Endoscopic images after ESD showed a flat ulcer. $\mathbf{h}$, I No residual tumor or stenosis was observed endoscopically at 4 months after treatment.

No residual tumor or stenosis was observed endoscopically at 4 months after treatment ( $>$ Fig. 2 h, i).

Surgical resection is usually recommended for symptomatic esophageal lymphangioma larger than $2 \mathrm{~cm}$ [2]. To the best of our knowledge, this is the largest esophageal lymphangioma that has been completely and successfully removed using ESD.

Endoscopy_UCTN_Code_TTT_1AO_2AG
Competing interests

None

The authors

Luli Hu, Kuang-I Fu, Biguang Tuo, Lianjun Di, Xuemei Liu, Kui Zhao, Huichao Wu

Department of Gastroenterology, Affiliated Hospital, Zunyi Medical College, Zunyi, China
Corresponding author

\section{Huichao Wu, MD}

Department of Gastroenterology, Affiliated Hospital, Zunyi Medical College, Zunyi 563003, China

Fax: +86-851-28609205

wuhuichao985@163.com

\section{Acknowledgment}

The authors thank Prof. Kuang-I Fu for editorial assistance on this paper. 

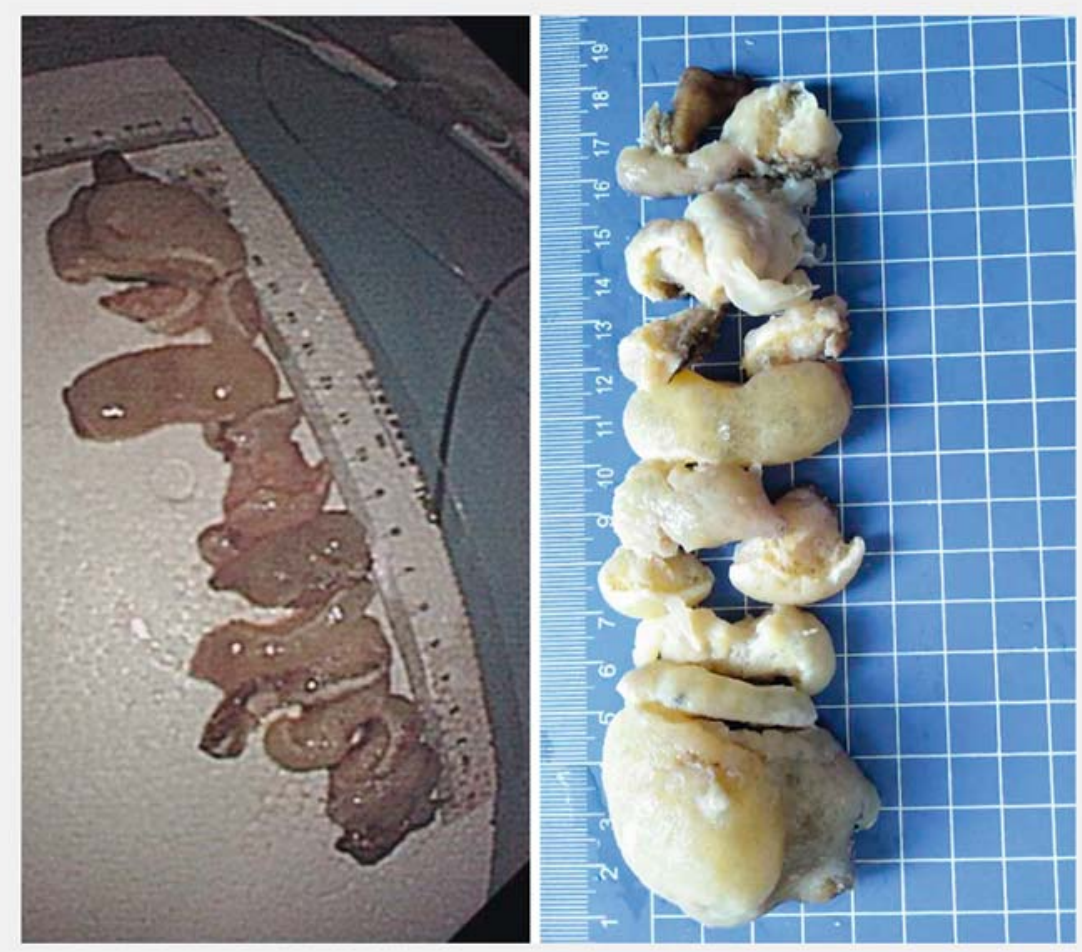

Fig. 3 Macroscopic appearance of the tumor.
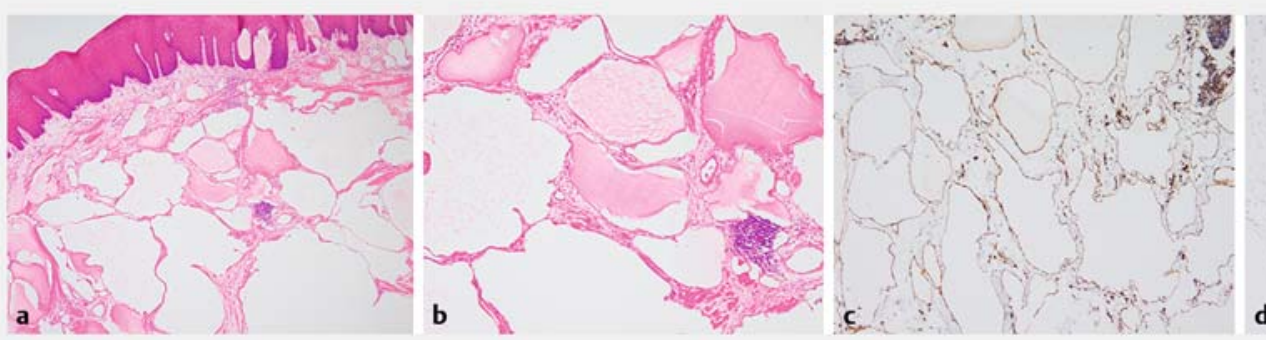

- Fig. 4 Histology of the resected specimen showed irregularly dilated lymphatic vessels beneath squamous epithelium and the submucosa, with lymphoid fluid in the lumen. Hematoxylin and eosin: $\mathbf{a} \times 40$ magnification; $\mathbf{b} \times 100$ magnification. $\mathbf{c}$ Lymphatic epithelial cells were stained with CD31 antibody $(\times 100)$. $\mathbf{d}$ The resected mass was positive for D2-40 $(\times 100)$.

\section{References}

[1] Arashiro M, Satoh K, Osawa H et al. Endoscopic submucosal dissection of esophageal lymphangioma: a case report with a review of the literature. Clin J Gastroenterol 2010; 3: $140-143$

[2] Luo D, Ye L, Wu W et al. Huge lymphangioma of the esophagus resected by endoscopic piecemeal mucosal resection. Case Rep Med 2017; 2017: 5747560

\section{Bibliography}

DOI https://doi.org/10.1055/a-0605-2443

Published online: 9.5.2018

Endoscopy 2018; 50: E181-E183

(c) Georg Thieme Verlag KG

Stuttgart · New York

ISSN 0013-726X

\section{ENDOSCOPY E-VIDEOS}

https://eref.thieme.de/e-videos

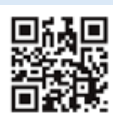

Endoscopy E-Videos is a free access online section, reporting on interesting cases and new techniques in gastroenterological endoscopy. All papers include a high quality video and all contributions are freely accessible online.

This section has its own submission website at https://mc.manuscriptcentral.com/e-videos 